\title{
User Flexibility Aware Price Policy Synthesis for Smart Grids
}

\author{
Toni Mancini, Federico Mari, Igor Melatti, \\ Ivano Salvo, and Enrico Tronci \\ Sapienza University of Rome, Italy \\ Roma, Italy
}

\author{
Jorn Klaas Gruber, Barry Hayes, \\ and Milan Prodanovic \\ Instituto IMDEA Energía, Spain
}

Lars Elmegaard
SEAS-NVE, Denmark

\begin{abstract}
In order to optimally manage a modern electricity distribution network, peaks in residential users demand should be avoided, as this can reduce energy and network asset management costs. Furthermore, this must be done without compressing residential users demand. To this aim, in a demand response setting, residential users are given a price policy, which economically motivates them to shift their loads in order to achieve this goal. However, if the price policy for all users is similar, this demand response may result in simply shifting the demand peaks (peak rebound), leaving the problem unsolved. In this paper we propose a novel methodology which i) for each network substation $s$, automatically computes the desired power profile to be kept in order to optimally manage the network itself; ii) for each network substation $s$, automatically synthesizes individualized price policies for residential users connected to $s$, so that $s$ is kept at the desired profile. Note that price policies individualization avoids the peak rebound problem, as different users have different low tariff areas. Furthermore, our methodology measures the flexibility of a residential user as the capacity needed by a home energy storage system (e.g., a battery) to always follow the given price policy, thus mitigating residential users discomfort. We show the feasibility of our approach on a realistic scenario taken from an existing medium voltage Danish distribution network.
\end{abstract}

\section{INTRODUCTION}

In order to optimally manage a modern Electric Distribution Network (EDN), a Distribution System Operator (DSO), which is responsible for managing all aspects of the EDN, has to avoid peaks in residential users aggregated electrical energy demand, as such peaks increase costs for energy production and systems maintenance. This is due to the fact very high demand require increased use of expensive peaking power plants. Demand peaks can also reduce power equipment lifetimes, and in extreme cases, result in overloading and voltage issues in the networks [1].

However, demand peaks must be avoided without cutting (i.e., decreasing) the residential users demand. In fact, this would decrease the income not only for the DSO, but also to the energy retailer (which directly sells energy to residential users). Furthermore, residential users would not accept a solution which forces them to cut their needs. Thus, DSOs and energy retailers need to achieve peak shaving, i.e. to (minimally) re-distribute (load shifting) the residential users power demand in a way that peaks are avoided while not compressing the overall residential users power demand. That is, the residential users power demand, considered on a long enough period (e.g., one month or one year), must be redistributed (i.e., shifted), but the total energy demand

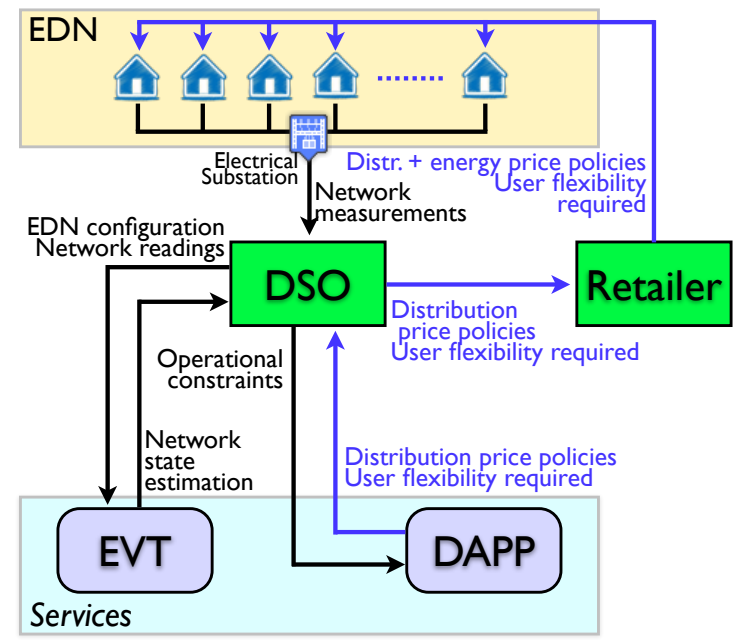

Fig. 1: The proposed services architecture. Black lines are for EDN related information, blue lines are for residential homes information.

must not decrease, as DSOs and energy retailers gains are based on how much energy they are able to sell. To this aim, in a Demand Response (DR) setting, the DSO and the energy retailer propose to residential users a price policy, which economically motivates them to shift their loads so that global peak shaving is achieved.

Unfortunately, such DR methodology has two main drawbacks: i) this may result in simply shifting the peaks to off-peak hours (peak rebound), leaving the problem unsolved [2], [3]; ii) only certain tasks (such as, dish washing, laundry, etc.) can be time shifted without creating discomfort to users. Others, energy eager, tasks such as cooking, heating, air conditioning cannot be significantly shifted and this limits the impact of incentive based DR schemata. As an consequence, residential users load shifting is very limited [4].

\section{A. Contribution}

In order to improve this state of the affairs and overcome the limitations of DR approaches, in this paper we introduce a novel methodology to improve EDN management (see Fig. 1). Namely, our methodology rests on two integrated services, and exploits the EDN hierarchy induced by EDN substations interconnection. The first service, which we call EDN Virtual Tomography (EVT) service, considers the whole EDN, detects possible violations of network constraints, and computes operational constraints on several EDN components, 
in order to improve EDN usage. Such constraints may be easily casted by the DSO as desired power profiles on each EDN substation $s$, thus defining the safety conditions for each $s$. Thus, peak shaving may be casted to the problem of keeping the aggregated residential users power demand on each $s$ below the power thresholds defined by the desired profile for $s$. To this aim, the other service, which we call Demand-Aware Price Policy (DAPP) service, motivates residential users load shifting, by considering each substation $s$ separately. This is done by computing, for each residential home $u$ connected to $s$, an individualized suggested power profile $P_{u}$ (that is, different users may get different power profiles), so that if all users follow their power profile then the operational constraints on $s$ are met. Each user $u$ is motivated to follow the suggested power profile $P_{u}$ by an individualized price policy based on $P_{u}$. Note that considering each substation separately allows us to reduce price policies computation complexity.

In the following, we describe EVT and DAPP, and outline how they mitigate the obstructions (peak rebound, residential users discomfort) discussed above.

1) EVT: The EVT service uses measurements of the local energy consumption and generation obtained at certain points in the EDN to compute voltages, currents and other physical values for locations in the EDN not directly accessible by sensors. Hence, this service offers information on the grid state from virtual sensors, by exploiting measurements of local energy consumption and generation, as well as the known EDN topology. The EVT service is intended to help the DSO to improve EDN operating conditions, through improved network observability, even in parts of the EDN where sensors are not available. Unexpected events (e.g., communication failures) can lead to the presence of bad data in measurements. For this reason, EVT is also able to detect and handle bad data.

2) DAPP: The main goal of DAPP is to optimize EDN operation at substation level, by performing peak shaving. In order to do this, DAPP individually motivates residential users to perform load shifting. This is achieved by automatically computing, with a given periodicity (one day in our experiments), an individualized power profile $P_{u}$ for each residential user $u$ connected to a given EDN substation $s$. Each power profile defines a low tariff area, so that the per-unit price of energy is low inside power profile $P_{u}$ and high outside. We designed DAPP so that its output has the following properties. First, depending on the home energy contract (i.e., on the maximum power which may be required at any time), a daily guaranteed amount of energy is always available at the low price. In this way, residential users demand is not compressed, and the price policies are generated in a fair way (based on home energy contract and on user needs). Second, as price policies are individualized, each residential user gets different off-peak hours. As a result, the peak rebound problem is avoided. Third, to mitigate residential users discomfort, which is the main obstruction for DR approaches, DAPP also outputs, for each residential user $u$, the flexibility which is required to $u$ in order to follow the price policy of $u$. Such flexibility is modeled as the minimum capacity of a virtual Energy Storage System (ESS) to be installed by $u$ in order to always follow the price policy and pay the low price. In this paper we only focus on such virtual ESSs, leaving as future work management of actual home ESSs. Furthermore, DAPP also outputs a witness charge/discharge plan for the virtual ESS, allowing $u$ to stay inside the low tariff area. This allows us to base the price policy contract for $u$ on a numerical evidence, thus mitigating users discomfort. Finally, if $u$ actually equips the home with an ESS (e.g., a battery), and uses an automatic approach (like, e.g., [5]) to drive it with the goal of minimizing the energy bill, then staying into the low tariff area becomes transparent to $u$, thus further reducing residential users discomfort.

This paper is organized as follows. We first describe the algorithms behind each service in our approach. Namely, Sects. II and III describe EVT and DAPP, respectively, by also formalizing (Sect. III-A) our notion of user flexibility. Finally, Section IV describes a meaningful and real-world scenario, on which our approach is evaluated. Namely, we show that our integrated methodology is able to achieve peak shaving without compressing users demand, and to compute residential users flexibility in order to mitigate users discomfort.

\section{B. Related Work}

The paper closest to ours is [6], where an integrated methodology has been presented to enforce EDN substations safety by proposing individualized price policies to residential users. However, the approach in [6] does not target users flexibility, as it outputs price policies which aims at staying close to user habits, without providing any measure of such closeness. This is instead the main goal of this paper, as the flexibility output by DAPP allows us to mitigate the users discomfort obstruction. Furthermore, w.r.t. the approach in [6], we also avoid to compress residential users demand. Finally, the experimental results we present here are based on an EDN with more residential users than the one used in [6].

Distribution networks have traditionally been designed as unidirectional links between transmission network bulk supply points and end-users. Typically they have been operated as passive systems, in which power flows are relatively easy to predict and manage. Recently, distribution networks have seen increasing penetrations of Distributed Energy Resources (DER), such as small to medium-sized renewable generators, demand-responsive loads, electric vehicles, devices with storage capability, heat pumps, etc. All relevant studies suggest that such trends towards more actively-managed distribution systems are set to continue, and that the integration of these technologies will lead to more frequent occurrences of problems in the distribution network, such as congestions and excessive voltage variations [7]. This has led to interest in adapting network management techniques, previously only used at the transmission level to distribution systems, such as State Estimation (SE) and short-term operational planning [8], [9], [10]. State estimation has been a standard feature of transmission network operation for several decades, where it is applied to improve the observability of the network, and reduce the impacts of noise and errors in system measurement data [11]. Recently, there has been significant research interest in developing state estimators specifically for use in EDNs [12], [13]. Distribution network state estimation is often applied as part of an advanced distribution management system, designed to monitor and optimise the energy flows and operation of active EDNs with significant DER [14], [9]. The EVT service developed in this paper employs state estimation to the EDN in order to accurately determine the grid state, 
and then uses this information to provide warnings, alarms and recommendations to the DSO.

The DAPP service is based on the DR approach, as it relies on price policies in order to motivate users to follow given power profiles. More in detail, price policies output by DAPP are based on the Inclining Block Rates (IBRs) (e.g., see [15]) approach, where usage beyond a given threshold entails an higher per-unit price. Moreover, as the IBR threshold varies with time, DAPP price policies also follow a Time of Usage (ToU) schema. A competing approach for DR is the Direct Load Control (DLC) approach (see, e.g., [16]), where the utility (DSO) remotely controls energy consumption by curtailing high-load household appliances. In this paper, we focus on residential users and we use a DR approach as DLC approaches typically cause discomfort to final users due to privacy issues [17]. We also point out that we focus on a price-based DR approach, instead of the traditional eventbased DR approach where the utility requests the shedding of load. Both DR and DLC are Demand Side Management (DSM) [18] methodologies, which are used to regulate EDN by acting, directly or indirectly, on end users. Note that indeed DAPP synthesizes price policies starting from system level formal specifications. See [19], [20], [21], [22], [23] for related work on synthesis from system level formal specifications, which however focuses on synthesis of controllers for hybrid systems. See instead [24], [25], [26], [27] for related work on verification of system level formal specifications. As for price policies computation, many works are based on distributed algorithms and architectures (e.g., as in [28]). On the contrary, DAPP is based on a centralized algorithm which computes all required price policies. A centralized (Neural Network based) approach similar to the one in DAPP is in [29]. Differently from [29], DAPP assigns a different price policy to each user in the same area (e.g., connected to the same substation), considers the case where users are producing energy, focuses on users flexibility and avoids demand compression, by using a Linear Programming (LP) based approach. Finally, we point out that the mechanism used by DAPP to encourage users to follow such a suggested behaviour is pricing, which is decided by the energy retailer based on economical as well as social considerations, as described, e.g., in [30].

\section{EDN VIRTUAL TOMOGRAPHY}

The EVT service is aimed at assisting the network operator (DSO) in the operation and management of "active" EDNs, i.e. distribution networks with significant DER. In most EDNs only few real-time measurements are taken due to technical and economic issues [10]. However, the EVT uses all available measurements, along with a detailed simulation model of the EDN to compute a wide range of physical values and estimate the state of the network [31]. The results of this SE can then be used to automatically generate warnings and alarms if a value approaches or exceeds its limits. The EVT service (Fig. 2) is comprised of two modules, or functions: (A) the estimation of the EDN state; and (B) the generation of warnings, alarms and advice for the DSO.

\section{A. State Estimation}

The inputs to the first module of the EVT are the static network parameters (bus and branch information), along with measurement data from the network (e.g. real-time recordings

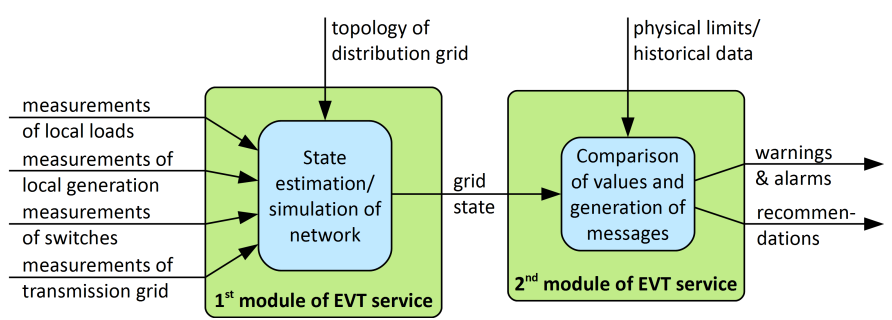

Fig. 2: Overview of the two modules of the EVT showing inputs and outputs.

active/reactive power injections from local load/generation the transmission system, active/reactive power flows). These data are fed to the state estimator, which identifies bad data, such as erroneous or missing values in the input measurements.

1) State Estimation Algorithm: The EDN state is expressed as the vector $\mathbf{x}$, containing the voltages and power angles at each node in the distribution system. To estimate $\mathbf{x}$, the set of measurements from the network, $\mathbf{z}$, is applied. The values in $\mathbf{z}$ can comprise of measurements of power/current injections or voltage magnitudes at system buses, measurements of active and reactive power flows in system branches, pseudomeasurements (i.e. estimates) of network quantities, or any combination of the above. This forms a set of over-determined, non-linear equations $\mathbf{z}=\mathbf{h}(\mathbf{x})-\mathbf{e}$, where $\mathbf{h}(\mathbf{x})$ are the power flow functions corresponding to each measurement in $\mathbf{z}$, and $\mathbf{e}$ is the vector of measurement errors. The most commonlyused approach to minimise the objective function $\mathbf{J}(\mathbf{x})$ is the Weighted Least Squares (WLS) method:

$$
\min _{x} \quad \mathbf{J}(\mathbf{x})=\overline{\mathbf{W}}(\mathbf{z}-\mathbf{h}(\mathbf{x}))^{2}
$$

where: $\overline{\mathbf{W}}$ is the measurement weight matrix. Each of the weights in $\overline{\mathbf{W}}$ are set according to the inverse of the variance of the corresponding metered system measurement. This allows the weights in $\overline{\mathbf{W}}$ to be adjusted so that the estimator gives more weight to input data points which are known to have greater accuracy. The minimisation in (1) is solved iteratively using the Newton-Raphson method.

2) Robust State Estimation: In the presence of gross input data errors (outlier values), conventional SE approaches can have computational issues, which can result in the minimisation in (1) becoming insoluble. The WLS estimator is particularly sensitive to outlier points, since the larger the residual, the larger an influence it has on the quadratic objective function. In order to improve the robustness of the SE in the presence of bad data, a number of authors have developed "robust" estimators [32], [33], which work by re-assigning the weights of $\overline{\mathbf{W}}$ as the SE minimisation is solved, so that the weights of suspected bad data (outlier) points are reduced in the calculation. In this paper, the equivalent weight function based on robust statistics theory in [32] is applied to create a "robust" WLS estimator.

3) Detection and Handling of Bad Data: The presence of bad data in the system measurement data set (e.g. due to measurement errors, or communication failures) can be detected by analysing the objective function $\mathbf{J}(\hat{\mathbf{x}})$, and the normalised residual vector given by $\mathbf{r}=\mathbf{z}-\mathbf{h}(\hat{\mathbf{x}})$. Statistical tests, such as the Chi-Squared test are applied to $\mathbf{J}(\hat{\mathbf{x}})$ and $\mathbf{r}$, allowing bad input data to be identified, provided there is sufficient redundancy in the measurement data set [10]. Estimates of demand and DER outputs, based on historical 
data are used to replace bad/missing measurements, and in cases where there is not enough measurement data available to accurately determine the state of the whole network [13].

\section{B. EVT Warnings, Alarms and Recommendations}

The second function of the EVT uses the estimated grid state to generate warnings and alarms, and give recommendations to the DSO, Fig. 2. This EVT module requires additional information on the physical limits of the EDN, as well as historical data in order to detect unusual values. Routines are set up to detect parameters which are trending out of control and provide warnings and alarms to the DSO. Appropriate corrective actions (e.g. network switching, load management) can be simulated off-line, allowing recommendations to be made to the DSO. In addition, EVT can be configured to provide general recommendations to the DSO for managing voltage, congestions and losses in the EDN. The overall status of the system is described by the EVT according to "operating state" categories outlined below, where the overall network state is described in one of three categories, which are used to direct DSO decisions around corrective actions.

- Normal: Normal network operation, no action required.

- Warning: Network operating close to allowed limits, potential for violation of system constraints. In this case, a warning is issued by the EVT, and recommendations are provided to the DSO, for instance, adapting network production/consumption to the network situation.

- Alarm: Network constraints are violated, generating alarms from the EVT. Such situations will require a reconfiguration of the network, or if this still cannot resolve the violation(s), direct load management or Distributed Generation (DG) curtailment is required to return network to a secure operating state.

\section{DEMAND-AwARE Price Policy}

In this section we describe our DAPP algorithm. To this aim, we first define our notion of residential users flexibility (Sect. III-A) and then we describe DAPP input and output (Sect. III-B). In Sect. III-C, a sketch of the DAPP underlying algorithm is also provided. Throughout such sections, the following notation is used. A time-slots set $T$ is a finite set of contiguous time-slots, all having the same duration. A power profile is a function $P: T \rightarrow \mathbb{R}$. A power profile tube (or region) is a pair of power profiles $\left(P_{l}, P_{h}\right)$ defined over the same $T$ s.t. $P_{l}(t) \leq P_{h}(t)$ for all $t \in T$. A power profile $P$ follows a power profile tube $\left(P_{l}, P_{h}\right)$ iff $P_{l}(t) \leq P(t) \leq P_{h}(t)$ for all $t \in T$. Finally, a $L P$ problem is a minimization problem over a set of linear inequalities (constraints) on real variables.

\section{A. Residential User Flexibility Model}

In our approach, each residential user $u$ is provided, with a given periodicity (every day in our experiments), with an individualized price policy to be followed. Such price policy is defined on the basis of an individualized power profile region $\left(P_{u, l}, P_{u, h}\right)$, which we also refer to as low tariff area. The resulting tariff for $u$, which we call DAPP tariff, is based on two prices for energy, the high price and the low price: if user $u$ needs power $P_{u}(t)$ in time-slot $t$, then $u$ will pay the low price if $P_{u}(t) \in\left[P_{u, l}(t), P_{u, h}(t)\right]$ (i.e., $P_{u}(t)$ is inside the low tariff area) and the high tariff otherwise. Note that the DAPP tariff is both IBR (two prices are used depending on user power demand), ToU (the low tariff area boundaries $P_{u, l}(t), P_{u, h}(t)$ vary with time) and individualized (the low tariff area boundaries vary with he user too). As an example, Fig. 5 shows the power upper bound $P_{u, h}$ together with the actual power demand $P_{u}(t)$ for a given user in the reference scenario we will use for our experimental evaluation. In the time-slot $t_{1}$ from $4 \mathrm{AM}$ to $5 \mathrm{AM}$, the user is outside the low tariff area (i.e., $P_{u}(t)>P_{u, h}(t)$ ), thus the high tariff will be applied, whilst in the previous time-slot $t_{2}$ from 3AM to 4AM the low tariff will be applied. In order to stay inside the low tariff area also in $t_{1}$, the user should be flexible and, as an example, move approximately $1.5 \mathrm{~kW}$ of power demand (i.e., $\left.P_{u}(t)-P_{u, h}(t)\right)$ from $t_{1}$ to $t_{2}$ (load shifting).

In this section, we want to provide a mathematical model for users flexibility. This will allow us to numerically compute the users flexibility required to follow a price policy, and base the user contract on such a computation. To this aim, we proceed as follows. We model flexibility of a residential user $u$ by means of a virtual (and ideal) ESS. As a consequence, we model the flexibility of $u$ by using a pair $\left(Q_{u}, R_{u}\right)$, where $Q_{u}$ is the ESS capacity (i.e., the maximum energy which may be stored, in $\mathrm{kWh}$ ) and $R_{u}$ is the ESS power rate (i.e., the maximum power which may be used from or saved into the ESS in a given time-slot). In the previous example, in order to perform the above described load shifting without changing user demand, it is sufficient to have $Q_{u}=1.5 \mathrm{kWh}$ and $R_{u}=1.5 \mathrm{~kW}$. Instead, in order to be able to stay in the low tariff area of Fig. 5 for the first 5 hours of the day (from midnight to $5 \mathrm{AM}$ ), the user flexibility required is $Q_{u}=$ $3.25 \mathrm{kWh}$, with a power rate $R_{u}=1.75 \mathrm{~kW}$. In fact, the first two hours need respectively 1.5 and $1.75 \mathrm{kWh}$ of energy from the ESS. Then, since $u$ needs, from 2AM to 4AM, less energy than the one allowed in low tariff, low-cost energy may be stored in those hours, in order to be used from 4AM to 5AM. We formalize the notion of flexibility sufficient to follow a given price policy as follows. Given a low tariff area $\left(P_{u, l}, P_{u, h}\right)$ on $T$ for user $u \in U$, we say that $\left(Q_{u}, R_{u}\right)$ is a flexibility sufficient to $u$ in order to follow $\left(P_{u, l}, P_{u, h}\right)$ iff there exists a plan for the charging/discharging actions on a ESS with capacity $Q_{u}$ and power rate $R_{u}$ allowing $u$ to stay inside the low tariff area. In formulas, there must exist a power profile $a_{u}$ on $T$ s.t. the following holds. (i) All values $a_{u}(t)$ may be interpreted as charge/discharge (if positive or negative, respectively) actions on the ESS modeling the flexibility of $u$. That is, $a_{u}(t) \in\left[-R_{u}, R_{u}\right]$ for all $t \in T$; (ii) The remaining capacity the ESS modeling the flexibility of $u$, as resulting from the charge/discharge actions, must always be a positive value less than the maximum capacity $Q_{u}$. That is, $0 \leq B_{u}(t) \leq Q_{u}$ for all $t \in T$, being $B_{u}(t+\tau)=B_{u}(t)+\tau a_{u}(t)$; (iii) By applying the $a_{u}$ to the power profile $P_{u}(t)$ of $u, u$ is always inside the low tariff area. That is, $P_{u, l}(t) \leq P_{u}(t)+a_{u}(t) \leq P_{u, h}(t)$ for all $t \in T$. Finally, the definition of flexibility required to follow a price policy is given by fixing the power rate $R_{u}$, as ESSs actually available on the market typically vary in the capacity and have a few available values for the power rate. Namely, given a low tariff area $\left(P_{u, l}, P_{u, h}\right)$ on $T$ and a power rate $R_{u}$ for user $u \in U$, we say that $\left(Q_{u}, R_{u}\right)$ is the flexibility required to $u$ in order to follow $\left(P_{u, l}, P_{u, h}\right)$ with power rate $R_{u}$ iff $Q_{u}$ is the minimum capacity among the flexibilities $\left(\tilde{Q}_{u}, R_{u}\right)$ which are sufficient to follow $\left(P_{u, l}, P_{u, h}\right)$. 


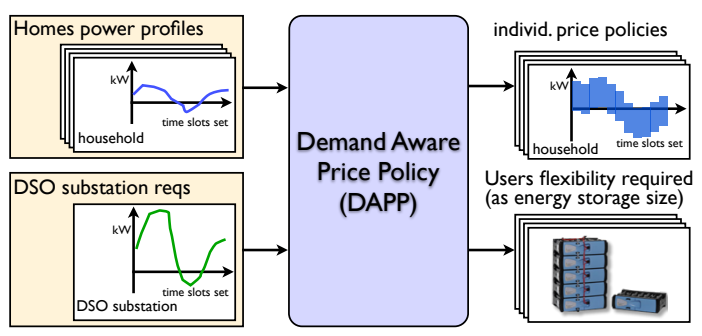

Fig. 3: DAPP input and output on a single DSO substation.

\section{B. DAPP Input and Output}

We may now describe in detail input and output for our DAPP algorithm (for a high-level view, see Fig. 3). Namely, DAPP requires the following input: (i) a set of homes $U$ connected to a substation $s$; (ii) a time-slots set $T$ (in our experiments, $T$ has a time span one day in the future); (iii) the desired power profile $P_{s}$ (in $\mathrm{kW}$ ) on $T$ for the substation $s$, as decided by the DSO on the basis of EVT output; (iv) for each $u \in U$, a forecast $d_{u}$ for the power profile of $u$ in $T$ (this may be computed on the basis of the energy usage of $u$ in the days preceding $T$ using, e.g., [34]); (v) for each user $u \in U$, the maximum power (in $\mathrm{kW}$ ) $C_{u} \in \mathbb{R}$ supported by the home main, as defined in the energy contract for electricity consumption and production (e.g., 3 or $6 \mathrm{~kW}$ ); (vi) for each user $u \in U$, the power rate $R_{u}$ (in $\mathrm{kW}$ ) for flexibility of $u$ (see Sect. III-A); (vii) the minimum energy (in $\mathrm{kWh}$ ) that each user $u$ must be guaranteed to be able to use in $T$, as a coefficient $\alpha$ multiplying the user energy contract $C_{u}$.

The output of DAPP is a set of individualized power profile regions (i.e., the low tariff areas) $\left(P_{u, l}, P_{u, h}\right)$ on $T$, for each residential user $u \in U$. By using the price for low and high tariff as decided from the energy retailer, such low tariff areas define the individualized price policies. Furthermore, for each user $u \in U$, the capacity $Q_{u}$ for the flexibility required to $u$ (see Sect. III-A) is also output by DAPP. Moreover, for each user $u \in U$, DAPP also outputs a charge/discharge plan $a_{u}$ on $T$ (see Sect. III-A), showing that the flexibility $\left(Q_{u}, R_{u}\right)$ is sufficient to follow the individualized price policy output by $u$. Finally, for each user $u \in U$, the DAPP collaborative profile for $u$ on $T$ is returned, as resulting from the application of plan $a_{u}$ to the input forecasted profile $d_{u}(t)$, i.e., $d_{u}(t)+a_{u}(t)$ for all $t \in T$. Note that, by definition of charge/discharge plan, the collaborative profile of a user $u$ is always inside the low tariff area for $u$.

\section{DAPP Algorithm}

DAPP algorithm consists in setting up a LP problem $L$ as follows. Decision variables of $L$ coincide with DAPP outputs. On such decision variables we define the linear constraints of $L$ so that: i) for each residential user $u \in U$, the DAPP collaborative profile for $u$ (as defined in Sect. III-B, must always be inside the low tariff area for $u$; ii) for each time-slot $t \in T$, if all users synchronise and use the maximum energy allowed in their low tariff areas, the resulting aggregated demand must be below the substation desired threshold (in order to achieve peak shaving); iii) for each residential user $u \in U$, the resulting low tariff area for $u$ must allow $u$ to use at least $\alpha C_{u} \mathrm{kWh}$ of energy through all $T$ (in order to avoid demand compression); and iv) for each residential user $u \in U$, the resulting maximum capacity $Q_{u}$ for the flexibility of $u$ must be proportional to the average daily demand of $u$ (fairness). Note that fairness is addressed by taking into account actual daily demand from users, so that greater flexibility will be required to users having greater demand. Once the LP problem has been created, it is solved by means of a LP solver (CPLEX in our experiments), and the required output is extracted from the solution returned by the LP solver.

\section{EXPERIMENTAL RESULTS}

In this section we show the technical evaluation of our approach by presenting the results we obtain on a reference scenario based on the medium voltage EDN actually used in a Danish village. Such EDN is a $10 \mathrm{kV}$ system with a mostly radial structure and a peak demand of $3.2 \mathrm{MW}, 77 \%$ of which is made up of residential users For such EDN, historical hour-byhour data on residential users energy consumption/production is available from 1st September 2013 to 31st August 2014 (12 months, which we refer to as reference period). Furthermore, for each home the energy contract (i.e., the maximum power which may be used at any time) is known. However, the aggregated user demand on EDN substations is currently too low, thus peak shaving is not an issue. In order to create a challenging scenario for DAPP, i.e., in which peak shaving is necessary, we proceed as follows. We choose the substation having the maximum number of homes equipped with heat pumps (which maximizes loads). This results in a substation $s$ with 62 homes connected to $s$. By using historical data on Plug-in Electric Vehicle (PEV) charging from the "Test-anEV" Danish project, we virtually equip each of such homes with a PEV, which increases the overall aggregated demand. Finally, we then create a pool of 186 homes, by replicating 2 times (plus the "original" copy) each of the aforementioned 62 homes, with the virtual PEV. In this way, for some periods of the year (namely, in Winter), peak shaving is necessary.

\section{A. EVT Evaluation}

Evaluation of is performed in one of the most critical loading scenarios on the given EDN, which take place in end January-beginning of February 2014. In this scenario, high demands and heavy EDN loading have resulted in the network operating close to the allowed limits, see Fig. 4 (left). Warnings are issued by the EVT, indicating that power flows are close to the allowed thermal limits (shown by the large flow arrows in Fig. 4, green arrows indicate active power flow, while blue arrows indicate reactive power flow), and alarms are issued for low voltages in most electrically distant parts of the network (the red areas in Fig. 4). As a consequence, EVT may advise that network switches should be re-configured to alter the power flows, or that load management could be carried out in certain parts of the EDN in order to avoid violation of operating constraints. For example, the DSO may define the desired power profile for the substation $s$ in the reference scenario to be at $90 \%$ of its nominal power (i.e., $400 \cdot 0.9=360$ $\mathrm{kVA}$ ) at certain peak time periods. The load shifting is enforced by the DAPP service, reducing the EDN demand peaks and avoiding the violation of line thermal constraints and voltage limits. Fig. 4 (right) shows the output from the EVT for the scenario when individualized price policies have been proposed to residential users at all applicable substations in the EDN. This reduced peak loading at network substations avoids the violations of system limits, and allows the EDN to maintain a normal operating state. 

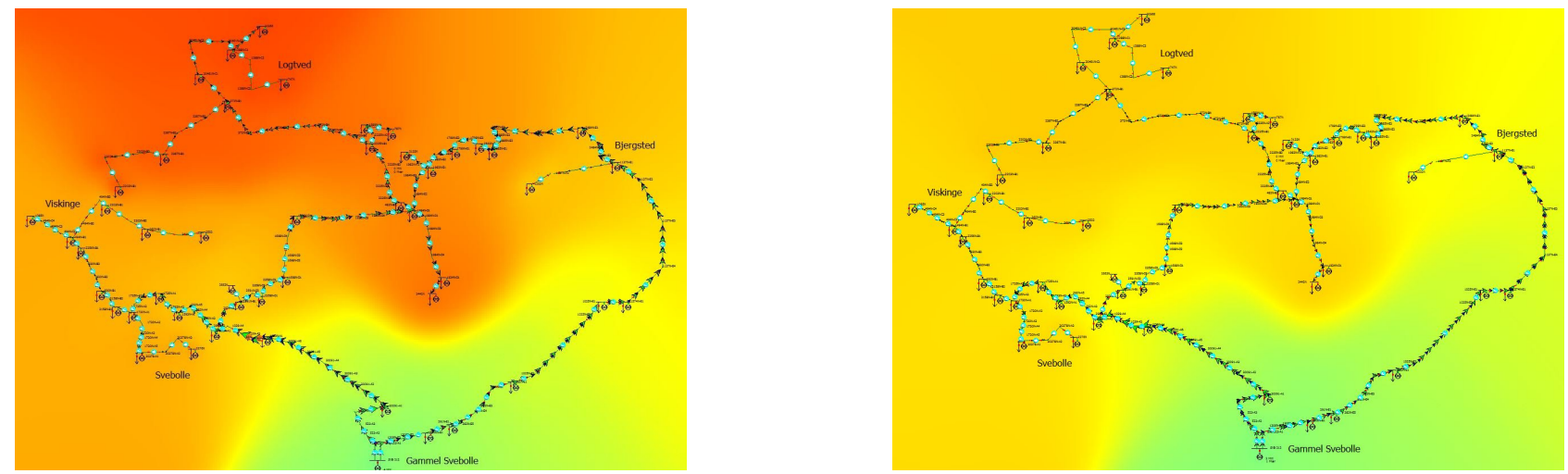

Fig. 4: Output from EVT when EDN substations are at peak demand without load management (left) and when EDN substations loading is managed as proposed by DAPP (right). In the first case, warnings and alarms are issued, indicating that the system is operating close to, or exceeding allowed limits. In the second case, the system is in a normal operating state.
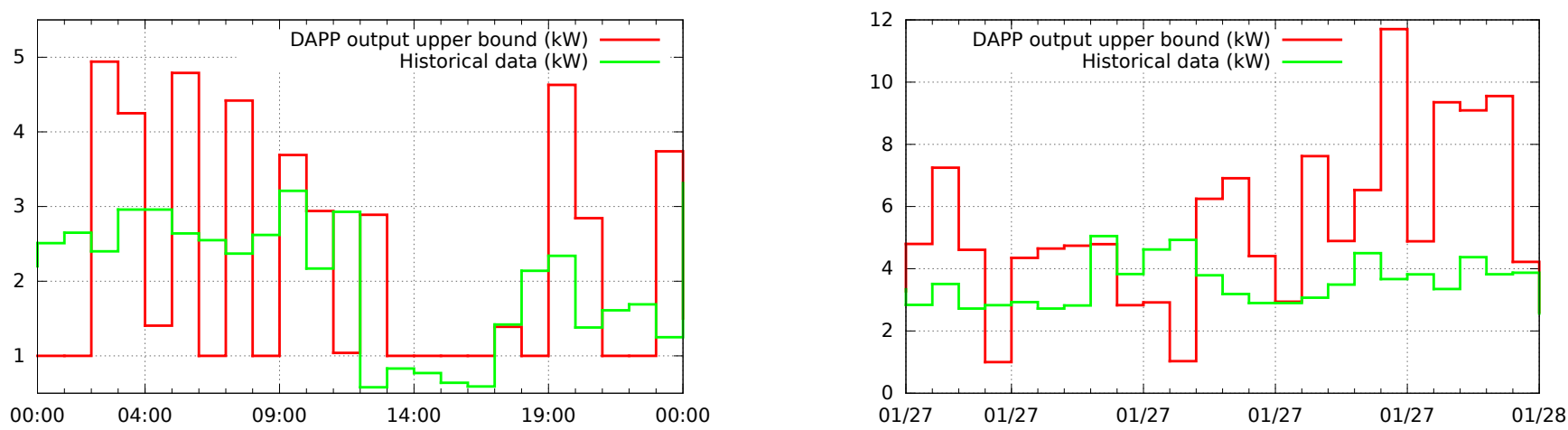

Fig. 5: Output of DAPP from two different residential users $u_{1}$ (left) and $u_{2}$ (right) perspective: individualized price policy upper bound on 27th January 2014 (inside peak period).

\section{B. DAPP Evaluation}

In this section we evaluate the DAPP service, in order to show that it is able to: i) keep EDN substations under ideal loading by performing peak shaving, enforcing the limits computed from EVT output; ii) achieve the above goal without compressing residential users demand; iii) compute the flexibility required to each residential user.

In order to perform the technical evaluation, we run DAPP 365 times (one for each day) from September 2013 to September 2014 on the substation defined $s$ in the reference scenario (see Sect. IV). In all such DAPP executions, we set the desired power profile $P_{s}$ to be always $90 \%$ of substation nominal power (i.e., $P_{s}(t)=360 \mathrm{~kW}$ ). This avoids synchronized peak rebounds among $s$ and other EDN substations, which would be possible if the $90 \%$ limit is set only at peak hours as suggested by EVT output, as described in Sect. IV-A. Moreover, as for users flexibility power rate, we fix $R_{u}=2 \mathrm{~kW}$ for all users, as this is one of the most common solutions for ESSs available. As a result, for each day DAPP is able to compute an individualized price policy for each of the 186 residential users in the reference scenario in at most 4 minutes (requiring 500 MB of RAM memory at most). This shows feasibility of DAPP from the computational resources perspective on the reference scenario. As an example, Fig. 5 shows the output low tariff areas (note that we focus on the price policies upper bound, as this is our tool to keep substations under ideal loading), together with the historical data, for two of the 186 homes in the most demanding day of the reference period. Finally,
DAPP also requires as input the forecast of the demand for the next day. We compute such forecast by taking, for each residential user $u$ and for each time-slot $t$, the average demand of $u$ in time-slot $t$ on the days preceding the next one.

Furthermore, if all users follow their individualized policies (e.g., by behaving as the collaborative power profile returned by DAPP), then peaks are indeed avoided, as it is shown in Fig. 6. Namely, Fig. 6 shows both the user power profile from historical data and the DAPP collaborative power profile, both aggregated on all 186 homes in the reference scenario. The left part of Fig. 6 shows such values on the whole reference period, whilst the right part zooms on the most demanding period in the reference period. As a result, while power demand from historical data may be even higher of the $120 \%$ of substation nominal profile, power demand from collaborative users are always under $90 \%$. This shows that DAPP is indeed able to enforce peak shaving, and thus EDN substations ideal loading, on the reference scenario.

Moreover, DAPP achieves such result without compressing the residential users demand, as shown in Fig. 7 (left). Namely, we show the difference between the cumulative residential users aggregated demand with and without DAPP (i.e., DAPP collaborative demand vs. historical demand), as a percentage of the cumulative aggregated energy demand without DAPP. That is, for each time-slot $t$ we consider the result of summing the aggregated demand on all time-slots preceding $t$ in the reference period, and we show the values for such percentage in the whole reference period of one year. As a result, we 

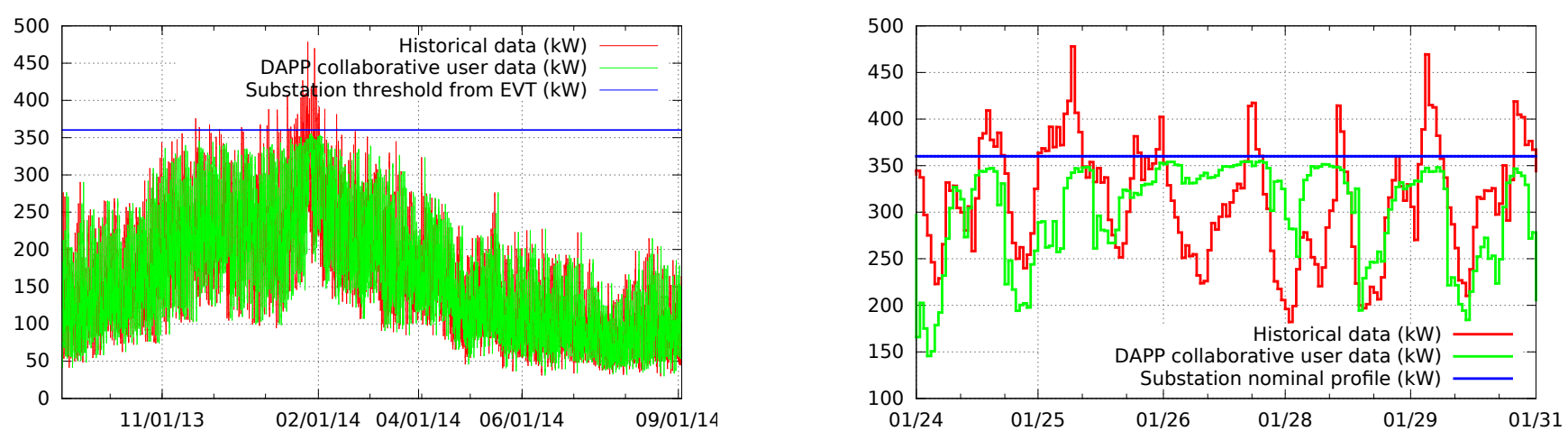

Fig. 6: DAPP output from the DSO perspective: peak shaving on the whole reference period (left) and from 24th January to 31 st January 2014 (right, peak period). Note that historical data follows the typical expected load (peaks in the evening, smaller peaks in the mornings).
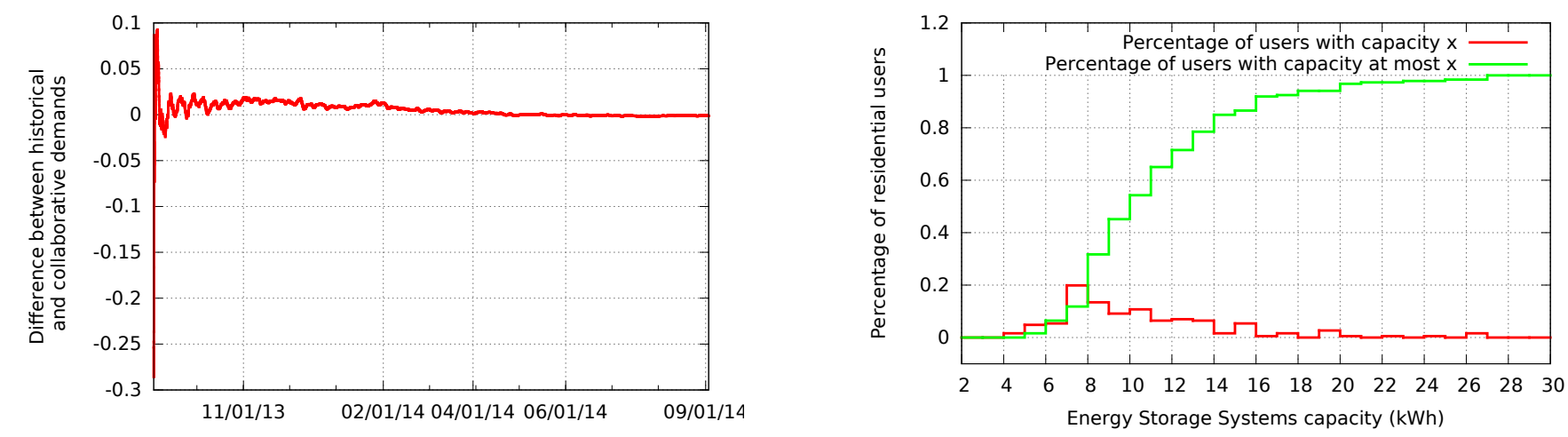

Fig. 7: DAPP output: demand is not compressed (left) and frequency distribution of users flexibility, in kWh (right).
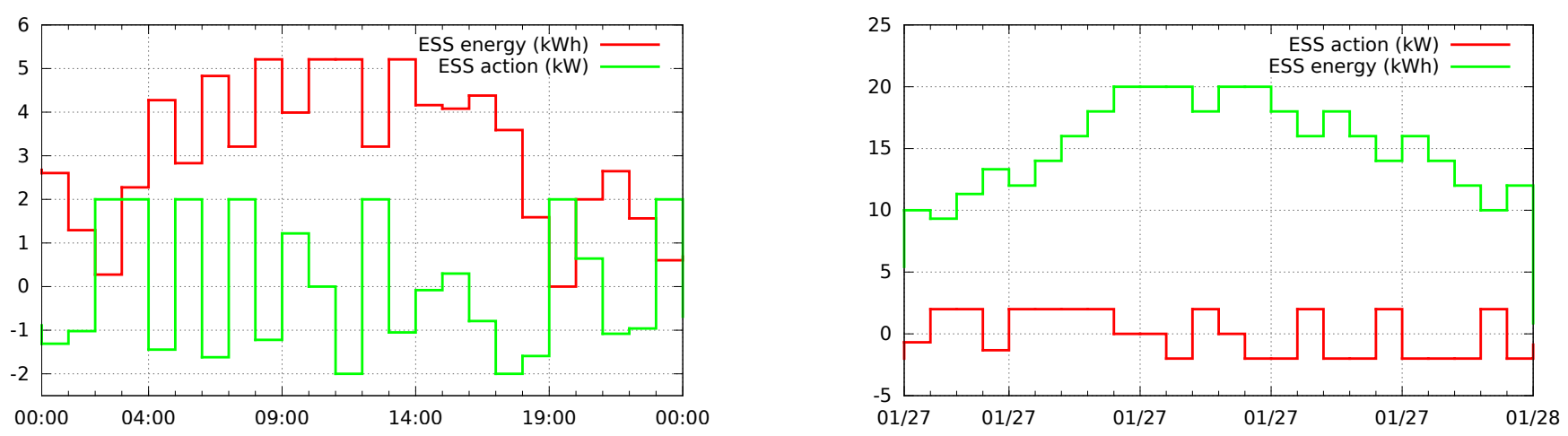

Fig. 8: Output of DAPP from residential users $u_{1}$ and $u_{2}$ perspective (see Fig. 5): charge/discharge plan for user flexibility ESS on 27th January 2014 (inside peak period).

may note that the average of positive values for the difference between demand with and without DAPP (i.e., in which demand is compressed) is $1 \%$, and never above $2 \%$ (excluding the first week of computation, where the difference may be $10 \%)$. Furthermore, at the end of the whole evaluation period, the difference is negative, thus the demand has been actually increased.

As for residential users flexibility, Fig. 7 (right) shows the flexibility required to residential users in the reference scenario in order to achieve the above results. Namely, we show, on the $x$ axis, the maximum ESS capacity (in $\mathrm{kWh}$ ), and on the $y$ axis the percentage of residential users. In such coordinates, two curves are shown: the first one shows the percentage of users for which the required maximum ESS capacity is in the corresponding $1 \mathrm{kWh}$ interval on the $x$ axis, whilst the second one shows the percentage of users for which the maximum capacity is at most the corresponding on the $x$ axis (i.e., the second curve is the integral of the first curve). As a result, we may note that the ESS needed capacity is less than $12 \mathrm{kWh}$ for nearly $80 \%$ of users, and less than $30 \mathrm{kWh}$ for all users.

Finally, Fig. 8 shows, for the same two residential users and the same day in Fig. 5 the charge/discharge plan output by DAPP for those residential users. By applying such plans, it is possible to always stay inside the low tariff area.

\section{CONClusions AND Future Work}

In this paper we presented a novel service-based methodology to improve EDN management. Namely, with our approach, 
the energy retailer and the DSO are able to sell as much energy as possible (as residential users demand is not compressed), while avoiding EDN overloading. To this aim, a DR approach is used, in which each user is provided with an individualized price policy. Finally, residential end users are also provided a measure of the flexibility needed in order to always stay inside the low tariff area defined by their price policies, thus lowering down their energy bills. Our approach is based on two integrated services: (i) EVT monitors the whole EDN, estimates the network state, and provides warnings, alarms and recommendations to the DSO. (ii) given an EDN substation $s$, DAPP proposes individualized price policies (based on individualized power profiles) to all end users connected to $s$, so that desired operational constraints on $s$ are met if each user follows the proposed power profile. We showed the feasibility of our approach on a medium voltage EDN in a Danish village. Namely, in the reference scenario, EVT was able to suggest to DSO operational constraints on each substation in order to reduce demand peaks and maintain the EDN within its operating limits. We showed that DAPP was able to motivate residential users to move their loads in order to (unknowingly) meet the operational constraints on $s$. This was accomplished by computing the flexibility required by each user in order to follow the individualized price policy, and providing an actual plan for load shifting, under the assumption that the flexibility is modeled as an ESS to be installed at home.

As future work we plan to further improve our services. Namely, EVT may be improved by refining methods for dealing with bad data and missing measurements in the input data. As for DAPP, we plan to also consider the case in which residential homes are actually equipped with cost-effective ESSs (whilst in this paper we only use ESSs to model users flexibility). In such scenario, we plan to design automatic control software able to control the home ESS in order to always follow price policies output by DAPP.

Acknowledgments: The research leading to these results has received funding from the EU Seventh Framework Programme (FP7/2007-2013) under grant agreement n. 317761 (SmartHG).

\section{REFERENCES}

[1] A. D. Hilshey, P. D. H. Hines, P. Rezaei, and J. R. Dowds, "Estimating the impact of electric vehicle smart charging on distribution transformer aging," IEEE Trans. Smart Grid, vol. 4, no. 2, pp. 905-913, 2013.

[2] S. Kishore and L. V. Snyder, "Control mechanisms for residential electricity demand in smartgrids," in Proc. of SmartGridComm, 2010

[3] A. Mishra, D. Irwin, P. Shenoy, J. Kurose, and T. Zhu, "Smartcharge: Cutting the electricity bill in smart homes with energy storage," in Proc. of e-Energy, 2012,

[4] F. Economics and S. First, "Demand side response in the domestic sector - a literature review of major trials," UK Department of Energy and Climate Change, Tech. Rep., August 2012.

[5] A. H. M. Rad and A. Leon-Garcia, "Optimal residential load control with price prediction in real-time electricity pricing environments," IEEE Trans. Smart Grid, vol. 1, no. 2, pp. 120-133, 2010.

[6] T. Mancini, F. Mari, I. Melatti, I. Salvo, E. Tronci, J. K. Gruber, B. Hayes, M. Prodanovic, and L. Elmegaard, "Demand-aware price policy synthesis and verification services for smart grids," in Proc SmartGridComm, 2014

[7] A. Keane, L. Ochoa, C. Borges, G. Ault, A. Alarcon-Rodriguez, R. Currie, F. Pilo, C. Dent, and G. Harrison, "State-of-the-art techniques and challenges ahead for distributed generation planning and optimization," IEEE Trans. Power Syst., vol. 28, no. 2, 2013.
[8] A. Meliopoulos, E. Polymeneas, Z. Tan, R. Huang, and D. Zhao, "Advanced distribution management system," IEEE Trans. Smart Grid, vol. 4, no. 4, pp. 2109-2117, Dec 2013.

[9] B. Hayes, I. Hernando-Gil, A. Collin, G. Harrison, and S. Djokic, "Optimal power flow for maximizing network benefits from demandside management," IEEE Trans. Power Syst., vol. 29, n. 4, 2014.

[10] B. P. Hayes and M. Prodanovic, "State estimation techniques for electric power distribution systems," in Proc. of EMS, 2014.

[11] Y.-F. Huang, S. Werner, J. Huang, N. Kashyap, and V. Gupta, "State estimation in electric power grids: Meeting new challenges presented by the requirements of the future grid," Signal Processing Magazine, IEEE, vol. 29, no. 5, pp. 33-43, 2012.

[12] E. Manitsas, R. Singh, B. Pal, and G. Strbac, "Distribution system state estimation using an artificial neural network approach for pseudo measurement modeling," IEEE Trans. Power Syst., vol. 27, n. 4, 2012.

[13] B. Hayes, J. Gruber, and M. Prodanovic, "A closed-loop state estimation tool for MV network monitoring and operation," IEEE Trans. Smart Grid, vol. PP, pp. 1-1, 2015, DOI: 10.1109/TSG.2014.2378035.

[14] D. Haughton and G. Heydt, "A linear state estimation formulation for smart distribution systems," IEEE Trans. Pow. Syst., vol. 28,n. 2, 2013.

[15] P. Reiss and M. White, "Household electricity demand, revisited,", Rev. Econ. Studies, vol. 72, no. 3, pp. 853-883, July 2005.

[16] P. J. Douglass, R. Garcia-Valle, P. Nyeng, J. Østergaard, and M. Togeby, "Smart demand for frequency regulation: Experimental results," IEEE Trans. Smart Grid, vol. 4, no. 3, pp. 1713-1720, 2013.

[17] “ADVANCED project deliverables: http://www.advancedfp7.eu/," 2015

[18] M. C. Vlot, J. D. Knigge, and J. G. Slootweg, "Economical regulation power through load shifting with smart energy appliances," IEEE Trans. Smart Grid, vol. 4, no. 3, pp. 1705-1712, 2013.

[19] F. Mari, I. Melatti, I. Salvo, and E. Tronci, "Model based synthesis of control software from system level formal specifications," $A C M$ TOSEM, vol. 23, no. 1, 2014.

[20] V. Alimguzhin, F. Mari, I. Melatti, I. Salvo, and E. Tronci, "On-the-fly control software synthesis," in Proc. of SPIN 2013, LNCS 7976

[21] _ - "A map-reduce parallel approach to automatic synthesis of control software," in Proc. of SPIN 2013, LNCS 7976

[22] _ " "On model based synthesis of embedded control software," in Proc. of EMSOFT, 2012

[23] _ "Automatic control software synthesis for quantized discrete time hybrid systems," in Proc. of CDC 2012.

[24] T. Mancini, F. Mari, A. Massini, I. Melatti, and E. Tronci, "System level formal verification via distributed multi-core hardware in the loop simulation," in Proc. of PDP, 2014, pp. 734-742.

[25] _ - "Anytime system level verification via random exhaustive hardware in the loop simulation," in Proc. of DSD, 2014, pp. 236-245.

[26] _ "Sylvaas: System level formal verification as a service," in Proc. of PDP, 2015, pp. 476-483.

[27] T. Mancini, F. Mari, A. Massini, I. Melatti, F. Merli, and E. Tronci, "System level formal verification via model checking driven simulation," in Proc. of CAV, 2013, LNCS 8044

[28] Z. Wang and L. Wang, "Adaptive negotiation agent for facilitating bi-directional energy trading between smart building and utility grid," IEEE Trans. Smart Grid, vol. 4, no. 2, pp. 702-710, 2013.

[29] N. Kunwar, Y. K., and R. Kumar, "Area-load based pricing in dsm through ann and heuristic scheduling," IEEE Trans. Smart Grid, vol. 4, no. 3, pp. 1275-1281, 2013

[30] P. R. Thimmapuram and J. Kim, "Consumers' price elasticity of demand modeling with economic effects on electricity markets using an agent-based model," IEEE Trans. Smart Grid, vol. 4, n. 1, 2013.

[31] B. Hayes and M. Prodanovic, "Short-term operational planning and state estimation in power distribution networks," in CIRED 2014

[32] J. Wu, Y. He, and N. Jenkins, "A robust state estimator for medium voltage distribution networks," IEEE Trans Pow Syst, vol.28, n.2,2013

[33] B. Hayes and M. Prodanovic, "A comparison of MV distribution system state estimation methods using field data," in IEEE PES, 2015.

[34] L. de Castro and P. Cramton, "Prediction markets for electricity demand," in Proc. of CCC, 2012 